\title{
Saúde Mental nas Escolas Médicas: Trabalhando com Percepções de Acadêmicos de Medicina
}

\author{
Cely Carolyne Pontes Morcerf ${ }^{1}$ (D) , Pedro Hernán Cabello Acero $^{2}$ iD \\ Universidade do Grande Rio - UNIGRANRIO, Rio de Janeiro, RJ, Brasil
}

\begin{abstract}
Resumo: A figura do louco, moldada como personagem causador de desordem, associada a temor e periculosidade levou à exclusão do convívio social, com expulsão de cidades e institucionalização de pessoas diagnosticadas com transtornos da mente durante a Idade Média. No século XVIII, o hospital entra como símbolo terapêutico e o médico se torna o responsável pela liberdade, tratamento e isolamento desses indivíduos. A rejeição do convívio social desses pacientes enraíza estigmas e preconceitos que são mantidos no meio médico atual e que necessitam de desconstrução na formação do estudante de medicina. $O$ presente trabalho buscar abrir debates e reflexões de percepções de acadêmicos sobre contato e abordagem de pacientes com transtornos mentais, ensino da saúde mental, trabalho da psiquiatria, terapias integrativas e possíveis impactos na prática clínica, direcionados para melhorias na formação do ensino em saúde. Trata-se de um estudo quali-quantitativo, de abordagem empírica, associado à revisão de literatura, com seleção de 39 artigos, que focam no ensino de saúde mental. Aplicação de questionário em 133 estudantes de medicina de uma universidade privada do Rio de Janeiro, que cursavam o primeiro, sexto período do curso e internato médico. As inferências foram feitas com auxílio do Software SPSS V.16. É destacado o sentimento de medo do estudante de medicina em relação ao primeiro contato com um paciente psiquiátrico, principalmente no início do curso $(p=0,000)$, a necessidade de ampliação do contato do estudante de medicina com a saúde mental $(p=0,04)$, o sentimento de despreparo para atender um portador de transtorno mental, falhas no referenciamento de pacientes para acompanhamento da psiquiatria, manutenção de visão hospitalocêntrica da assistência de saúde mental, carência de conhecimento e contato com terapias integrativas. É essencial que as faculdades de medicina ampliem a carga horária de ensino e contato da saúde mental, em seus diversos campos de atuação no SUS, garantam o suporte e a humanização da assistência, para a formação do médico generalista, e amparo dos estudantes quando os mesmos adoecem.
\end{abstract}

Palavras-chave: saúde mental, psiquiatria, educação médica

\section{Mental Health in Medical Schools: Working with the Perceptions of Medical Academics}

\begin{abstract}
The figure of the insane person, shaped as a character causing disorder, associated with fear and danger led to exclusion from social life, with expulsion from cities and institutionalization of people diagnosed with mental disorders during the Middle Ages. In the 18th century, the hospital became a therapeutic symbol and the doctor became responsible for the freedom, treatment and isolation of these individuals. The rejection of these patients' social life instills stigmas and prejudices that are maintained
\end{abstract}

1 Médica formada pela Universidade do Grande Rio (UNIGRANRIO), Barra da Tijuca; Professora convidada para orientações de trabalhos de conclusão de curso (TCC), Duque de Caxias. Rio de Janeiro, RJ, Brasil.E-mail: cely_carol@hotmail.com

2 Doutor em Ciências Biológicas pela Universidade de São Paulo (USP); Mestrado em Ciências Biológicas pela USP; Professor da disciplina de Saúde Coletiva da Universidade do Grande Rio (UNIGRANRIO). Rio de Janeiro, RJ, Brasil. E-mail: phca01@gmail.com

Submetido em: 21/02/2021. Primeira decisão editorial: 10/06/2021. Aceito em: 24/06/2021. 
in the current medical environment and that need to be deconstructed in the education of medical students. This work wants to open debates and reflections on academic perceptions about contact and approach to patients with mental disorders, mental health education, psychiatry work, integrative therapies and possible impacts on clinical practice, aimed at improving the training of health education. This is a quali-quantitative study, with an empirical approach, associated with a literature review with a selection of 39 articles, which focus on the teaching of mental health. Questionnaire application to 133 medical students from a private university in Rio de Janeiro, who were attending the first, sixth period of the course and medical internship. The inferences were made using the SPSS V.16 Software. The feeling of fear of the medical student in relation to the first contact with a psychiatric patient is highlighted, especially at the beginning of the course $(p=0.000)$, the need to expand the medical student's contact with mental health $(p=0.04)$, the feeling of unpreparedness to care for a patient with a mental disorder, failure to refer patients to psychiatry monitoring, maintenance of a hospital-centric view of mental health care, lack of knowledge and contact with integrative therapies. It is essential that medical schools expand the workload of teaching and contacting mental health, in its various fields of action in the health system, guaranteeing the support and humanization of care, for the training of general practitioners, and support for students when they get sick.

Keywords: mental health, psychiatry, medical education

\section{Introdução}

Os hospitais psiquiátricos, com caráter de longa permanência, se firmaram como alternativa para afastar pessoas que manifestavam condutas estranhas e fora do padrão de normalidade do meio em que viviam, assegurando assim um melhor e maior controle desses pacientes e um alívio para os demais membros da sociedade, que desconheciam informações sobre psicopatologia e outras teorias dos transtornos mentais, levando ao medo, ao estigma e ao desejo de viver distante de qualquer manifestação de loucura. Em paralelo a isso, o sujeito perdia sua individualidade e suas características pessoais, sendo os atos e comportamentos atribuídos exclusivamente à doença mental, com exposição à violência institucional (Martinhago \& Oliveira, 2015).

O desconhecimento da comunidade, o despreparo para reconhecimento de transtornos mentais, a negação e resistência frente à procura de acompanhamento em saúde mental se associam a percepções negativas, atitudes preconceituosas, estigmatização e afastamento de relações com essas pessoas, fruto de relações e percepções historicamente negativas dos transtornos mentais (Sakellari et al., 2014).

Um estudo realizado em 2012 abordou a percepção de estudantes universitários sobre a figura e o trabalho do psiquiatra e evidenciou uma ideia de profissionais competentes, porém com uma dificuldade de distinção em relação à atuação do psicólogo e do psiquiatra. A imagem de eficácia no tratamento farmacológico de transtornos mentais graves, porém pequena efetividade no tratamento da depressão foi destacada no estudo como reflexo do trabalho do psiquiatra moderno. A psiquiatria foi definida também como uma carreira de baixa popularidade, pela presença na visão dos estudantes do estigma associado ao campo da saúde mental e ao ambiente de trabalho do psiquiatra, definido como local de ampla carga emocional e estressante. Insatisfação no trabalho, baixo prestígio e renda inadequada foram pontuados como fatores que levam a ausência de interesse de estudantes de medicina em escolher a psiquiatria como área de especialização. A implementação de um maior número de métodos educativos, debates e intervenções práticas no campo da saúde mental podem ser grandes aliados no processo de rever e auxiliar na desconstrução do estereótipo e da percepção negativa do estudante de medicina frente ao paciente com transtorno mental (Firmin et al., 2012).

A saúde mental do acadêmico de medicina é estudada no Brasil desde a década de 50 pela alta prevalência de desencadeamento de transtornos mentais dentro do curso de formação médica. A universidade é a responsável pela formação da identidade profissional do estudante de medicina, processo que envolve, além do estudo da teoria e prática médica, complexo desenvolvimento de 
maturidade psicoafetiva, competências e habilidades ligadas à abordagem e solução de desafios, enfrentando situações adversas, angustiantes, dilemas éticos, morte e sofrimento. A educação médica começa a ser alvo de estudo no século $X X$, pela escassez de investimentos que englobem a moldagem da identidade do futuro profissional de saúde (Daltro \& Pondé, 2011).

Um grande número de estudantes que ingressam no ensino médico são adolescentes oriundos de escolas particulares, com bom nível sociocultural, que foram sempre acompanhados por familiares. Ao iniciar a vida universitária são recrutados à maturidade, porém, muitas vezes não conseguem responder a essa demanda e não encontram, na escola médica, um espaço onde possam recorrer e buscar auxílio, principalmente em relação ao comprometimento progressivo da saúde mental desses estudantes com a transição do ambiente do cursinho pré-vestibular para o ensino superior e com a evolução dos anos dentro da faculdade de medicina (Daltro \& Pondé, 2011; Marco, 2009).

No início do curso de Medicina, ocorre uma maior identificação do estudante com o paciente, o que é modificado durante o curso para uma maior identificação do estudante com a figura do médico. Assim, inicialmente o estudante possui empatia e sensibilidade frente à condição de saúde do paciente, buscando uma melhor forma de tratamento e abordagem daquele indivíduo. No decorrer do curso, essa sensibilidade e empatia é diminuída com o crescimento de mecanismos de defesas psíquicos para autoproteção, como defesas do ego, intelectualização e negação. Tais mecanismos podem levar a comportamentos de rigidez, levando à frieza, arrogância e distanciamento na relação médicopaciente (Marco, 2009; Mascia et al., 2009).

Dificuldades maiores são encontradas no impacto do contato com a morte e o transtorno mental. Os treinamentos em Saúde Mental e Cuidados Paliativos devem ser evidenciados e enfatizados durante a graduação, sendo necessário também um investimento maior em assistência psiquiátrica e psicopedagógica aos acadêmicos de medicina. Todos os esforços na melhoria da relação médico-paciente e da abordagem de temas angustiantes e desafiadores como a terminalidade da vida e o transtorno mental são essenciais para a formação de médicos generalistas competentes, éticos, com valores de responsabilidade social e livres de percepções errôneas, preconceituosas e estigmatizantes de pacientes com transtornos mentais, assim como o acolhimento de colegas acadêmicos de medicina que iniciam quadro de transtorno mental dentro da graduação. Os investimentos em uma educação médica com ênfase na formação do futuro médico com base na visão de ser humano biopsicossocial também é necessário para a identificação, orientação e abordagem do estudante de medicina com a saúde mental fragilizada, que necessita de uma intervenção precoce e de um olhar diferenciado para conseguir prosseguir sua formação com qualidade de vida e com o tratamento adequado (Mascia et al., 2009).

Sentimentos de repulsa e temor em relação ao contato com o paciente com transtorno mental, associados à imagem de periculosidade, violência e desordem levam à marginalização e ao abandono da preocupação com muitos aspectos da assistência e qualidade de vida do paciente com transtorno mental por estudantes da área de saúde. A história da loucura, baseada na psiquiatria tradicional, construiu uma ideia de loucura que deve ser excluída por perturbar a ordem pública, sendo de responsabilidade das autoridades o controle e a punição desses pacientes psiquiátricos, com atos de violência para controlar as manifestações de loucura percebidas como irreversíveis e muitas vezes até contagiosas aos que mantém contato e relações pessoais com o paciente. É necessária uma ampliação de espaços de ensino prático e vivências em saúde mental, com uma reaproximação do estudante da área de saúde do contato, da vida e do tratamento do paciente com transtorno mental, associado a um debate sobre os estigmas e as percepções negativas historicamente criadas desde a época da Idade Média, e ainda presentes nessa área, estimulando a interação e o aprendizado humano, responsável pelo cuidado da saúde física e mental do paciente. Assim, é essencial que atividades práticas em saúde mental e interações com pacientes portadores de transtornos mentais se relacionem a momentos de reflexão e 
discussão sobre cenários em que ocorre exclusão e marginalização do paciente com transtorno mental (Barcelos et al., 2010).

Relações entre estudantes, resultantes de situações cotidianas não contempladas no currículo estruturado, formam conjuntos de experiências com valores e atitudes, compondo o currículo oculto, que pode contribuir para a desumanização na graduação médica. Este currículo oculto possui aspecto deletério e têm reflexos na sobrecarga de acadêmicos, porém é uma via de avaliação do impacto no processo de ensino e aprendizado, reproduzindo valores de relações de poder presentes nas escolas médicas, influenciando nos valores de formação do futuro médico com base em redes hegemônicas e interações complexas moldadas durante o avançar dos ciclos até o fim do internato médico. Assim, o currículo oculto pode ser trabalhado, e os desafios encontrados no estudo de relações interpessoais, usados como instrumento de desenvolvimento das atividades de aprendizagem de saúde mental nas escolas médicas. Podem ser trabalhados de forma a estimular melhorias na qualidade de atendimento aos pacientes com transtornos mentais, principalmente nos estágios de psiquiatria, e ao entendimento da complexa rede social e familiar em que o paciente está inserido, respeitando a individualidade de cada caso e evitando juízos de valor e preconceitos sobre os pacientes que interfiram negativamente no acolhimento, no diagnóstico e nas formas de tratamento. A análise do currículo oculto pode ser utilizada para o entendimento de relações e problemas existentes, revertendo-se em melhorias na formação médica, com um estímulo a reflexões críticas dentro do processo de graduação. Tal abordagem pode ser inserida dentro de disciplinas como saúde e sociedade, saúde coletiva, saúde mental, psiquiatria e prática médica. $\mathrm{O}$ treinamento prático de enfrentamento, assim como incentivo a maiores abordagens em cenários de saúde mental, incluindo práticas integrativas e desenvolvimento de narrativas de estudantes a partir da interação com os pacientes, são imprescindíveis para a redução do estigma sobre o transtorno mental (Benedetto \& Gallian, 2018; Feijó et al., 2019; Santos et al., 2020; Touso \& Figueiredo, 2020).
As complexas relações interpessoais e hierárquicas, associadas à forte cobrança de perfeição e impossibilidade de erros, são alguns dos componentes que produzem adoecimento e desencadeiam transtornos mentais em estudantes de medicina. É crescente o número de estudos com objetivo de avaliar a prevalência de Transtornos Psiquiátricos Menores em estudantes do curso de Medicina, com um aumento de prevalência do início para o final do semestre. Tais transtornos comprometem a qualidade de vida e as relações sociais (Ferreira et al., 2016). Os agravantes ao adoecimento surgem de singularidades da formação médica, e os outros alunos, assim como professores e coordenadores do curso, não estão preparados para acolherem esses estudantes agora na figura de pacientes. Com isso são estigmatizados, rotulados como loucos, ignorados ou marginalizados dentro das relações de hierarquia da sala de aula. Tal comportamento reforça a exclusão do que é evidenciado como transtorno mental e os núcleos de apoio dentro da escola médica, quando funcionantes, não são acessados pelos estudantes por medo, incompreensão ou falta de referência e acompanhamento ao longo do curso. Ocorre então a perpetuação da marginalização e do silenciamento do que é caracterizado como fora dos padrões de normalidade, socialmente estipulados, no ambiente da universidade. Os estudantes de medicina que sofrem esse processo são ainda mais cobrados pela falsa visão de que o futuro médico deverá manter a estrutura, o equilíbrio e é o maior detentor e controlador da saúde. E de tantas patologias e sofrimentos que podem acometer um estudante de medicina, o transtorno mental é o maior alvo de preconceito (Dâmaso et al., 2019; Feijó et al., 2019).

As Diretrizes Curriculares Nacionais preconizam um ensino médico baseado no diálogo entre estudantes, universidades e sociedade, com foco na formação generalista e com relação de ensino e prática. A educação médica recebe iniciativas dos ministérios da Saúde e da Educação para o desenvolvimento de novas formas de ensino e aprendizado com mudanças de práticas pedagógicas tradicionais de faculdades, tentando aproximar da realidade de saúde e educação da 
comunidade e ao estímulo de métodos inovadores e ativos de ensino (Souza \& Lima, 2016; Gonçalves \& Benevides-Pereira, 2009).

O Sistema Único de Saúde contextualiza importantes espaços para a formação médica, norteando métodos inovadores de ensino e aprendizado na área de saúde. Uma vez que tradicionalmente a prática médica tem como base o encontro do médico com o paciente de uma forma individualizada, a criação de abordagens práticas de grupo quando inserida dentro do processo de formação é considerada prática inovadora por concentrar a atenção da atividade não apenas em um indivíduo, mas em um grupo, tendo como visão a coletividade, diferenciando da relação médicopaciente clássica e contribuindo como novo recurso de interação e aprendizado (Otanari et al., 2011).

Algumas vezes isso pode levar a uma não aceitação de determinados padrões de tratamento, sendo a explicação e orientação quanto ao diagnóstico e justificativa dos procedimentos e tratamentos a serem realizados um direito incontestável do paciente, independentemente de ter ou não uma síndrome psiquiátrica ou um determinado diagnóstico nosológico (Shackle, 1985).

A psiquiatria surgiu no século XIX, quando o hospital de loucos alienados deixaria de ser um depósito de indivíduos marginalizados para simbolizar um lugar para tratar a alienação, conferindo um status de doença à loucura. Assim, o hospital não perde a função de ser um ambiente de exclusão, porém a exclusão passou a ser medicalizada. Associado a isso, o doente mental diagnosticado pela psiquiatria não era reconhecido como alguém que detém saberes sobre seu próprio sofrimento, uma vez que a fundamentação ocorria em diagnósticos nosológicos, em que a classificação das doenças e o rótulo de ser um paciente com um transtorno mental distanciavam o processo de diagnóstico e tratamento da investigação relacionada ao sofrimento psíquico em sua complexidade (Lima et al., 2010).

O estudo da psiquiatria durante o internato médico, em determinadas faculdades, objetiva capacitar o acadêmico do curso de Medicina com conceitos de saúde mental e dos distúrbios psiquiátricos prevalentes na população brasileira, além de possibilitar o desenvolvimento de práticas interdisciplinares e comunitárias em saúde mental, buscando uma dissociação dos modelos da psiquiatria tradicional hospitalocêntrica. É essencial o desenvolvimento de competências e habilidades, além de um maior entendimento de conceitos em saúde mental que possibilitem uma abordagem e tratamento de um paciente com transtorno mental, com um enfoque generalista, evitando o subdiagnóstico e a desvalorização da necessidade de abordagens diferenciadas para determinados pacientes da psiquiatria (Paulin \& Poças, 2009; Cyrino \& Toralles-Pereira, 2004).

A figura do psiquiatra representa o profissional da área de saúde mental que tradicionalmente melhor associa aspectos biológicos e psicossociais, porém suas ações necessitam de recursos e técnicas para condutas que ainda não possuem clareza e elucidação suficiente, estando envoltas por limitações científicas e tal lacuna deve ser preenchida por treinamentos e uma educação médica continuada, visando a diminuição de incertezas em relação às condutas em psiquiatria. A pesquisa também é uma área importante dentro da saúde mental pela existência de grandes desafios e lacunas sobre temas, conceitos e abordagens em psiquiatria, principalmente no período atual de transição com o aumento de locais de assistência em saúde mental e de profissionais de saúde compondo a equipe multidisciplinar no tratamento de pacientes com transtornos mentais (Camargo \& Contel, 2004).

Recursos dinâmicos podem ser utilizados dentro de disciplinas e espaços extracurriculares em psiquiatria dentro da formação médica, como o uso de música, expressividade corporal, exploração do espaço físico da sala de aula, leitura de cartas, com recursos audiovisuais, entre outros, objetivando sensibilizar e levar a uma reflexão maior sobre conceitos e temas em saúde mental. Vivências com a utilização de metodologias diversificadas em cenários práticos, ativas e participativas auxiliam na discussão e sedimentação de conceitos em saúde mental, resultando em contribuições para a formação de futuros médicos generalistas com uma abordagem holística do paciente psiquiátrico, livre de percepções negativas e estereótipos. A abordagem 
através de metodologias diferenciadas do modelo de ensino tradicional podem ser um caminho para uma transformação efetiva do olhar em saúde mental (Soares et al., 2013).

Apesar de a ampliação de uma equipe de saúde mental, formada por diversos profissionais da área, e de o processo de reforma junto à consolidação e ao desenvolvimento do programa de saúde mental do SUS evidenciarem a importância do trabalho em equipes de saúde mental, alguns trabalhos mostram que a maioria dos cursos da área de saúde, durante o período de graduação, possui contato e diálogos restritos. Tal fato leva a uma marcada fragmentação de saberes que muitas vezes é transferida para a atuação profissional, levando a conflitos internos da equipe multiprofissional em saúde mental, prejudicando a unidade e o trabalho conjunto rumo à superação de barreiras e problemas existentes na continuidade das ações antimanicomiais e promotoras de saúde. Assim, atividades com ações interdisciplinares são tidas como conflituosas, como um desafio. Porém a integração profissional, desde a graduação até a atuação nas equipes de saúde mental e da atenção básica, exercita o pensamento complexo correspondente à base do paradigma da atenção psicossocial (Pereira et al., 2012; Castro \& Silva, 2002; Araruna et al., 2012; Amarante \& Torres, 2001).

O modelo positivista de ensino, que estratifica e fragmenta o conhecimento, ainda se encontra presente na educação de profissionais de saúde e nos campos de trabalho e é evidenciado por discursos de indivíduos que valorizam a especialização e o trabalho individualizado em detrimento da interação como uma unidade de saúde mental construída pela participação conjunta da equipe. A persistência de discursos dessa natureza entre profissionais de saúde reflete uma carência de abordagem dessa temática dentro das universidades e no espaço de formação e capacitação em saúde mental. Desde a graduação, a carga horária curricular direcionada à saúde mental em cursos de graduação e especialização é insatisfatória, com um maior foco teórico e expositivo em detrimento de vivências práticas, debates e reflexões em conjunto com ênfase em aspectos psicossociais (Amarante \& Torres, 2001; Alverga \& Dimeinstein, 2006).
A reestruturação da assistência psiquiátrica busca estimular cuidados comunitários com uma participação ativa da população, utilizando uma rede de saúde descentralizada e composta por um trabalho conjunto da equipe de saúde mental. Porém o apoio deficiente e a falta de incentivo a melhorias de condições de atendimento e salários dos profissionais de saúde mental podem ser considerados um dos fatores que contribuíram para a visão externa de uma área desvalorizada e inferior às outras áreas de atuação da medicina e da saúde em geral, além de divulgar um modelo falho e pouco organizado de atendimento em saúde mental descentralizado, levando à persistência da percepção de membros da sociedade da figura do hospital e do manicômio como melhor opção resolutiva para controle e tratamento de transtornos mentais (Ferreira-Furegato et al., 2010).

As representações sociais de acadêmicos da área de saúde estão fortemente ligadas à forma como será realizada a abordagem e o tratamento do paciente em sofrimento psíquico. Um estudo qualitativo com espaço amostral de 480 universitários dos cursos de Medicina, Enfermagem e Psicologia analisou atitudes e representações desses acadêmicos em relação à Reforma Psiquiátrica e ao paciente com transtorno mental. O trabalho teve como resultado uma grande imagem do paciente associada à exclusão e ao temor do contato com esse indivíduo, refletindo uma presença forte do paradigma biomédico, apesar dos avanços da luta antimanicomial e do programa de saúde mental (Sousa et al., 2016).

A realidade que envolve o estudante da graduação influencia diretamente a percepção de temas em saúde mental e da imagem do paciente em sofrimento psíquico. Assim, a representação social é uma interpretação das vivências, das experiências e do contato que foi realizado com esse grupo social, resultando em ideias estruturadas sobre temas específicos. Com isso, percepções de estudantes de medicina e outros cursos da área de saúde são de extrema importância para o entendimento de condutas e da prática realizada por esses futuros profissionais de saúde ao abordarem um paciente psiquiátrico (Sousa et al., 2016). 
Crenças e hábitos culturais são fatores que influenciam amplamente o estabelecimento e moldagem de percepções em saúde mental, que definirão muitas vezes a procura de tratamento ou a negação de um transtorno mental desenvolvido pelo sujeito, assim como o julgamento de outro indivíduo como paciente psiquiátrico. A não aceitação de diagnósticos em psiquiatria leva a um tratamento tardio, prejudicando a qualidade de vida e a saúde do paciente, seja ele adulto ou criança. Assim, um maior conhecimento sobre transtornos mentais e ações de educação em saúde sobre temas em psiquiatria são aliados na quebra de barreiras para a aceitação e o início do tratamento (Antunes et al., 2016; Brockelman \& Scheyett, 2015).

Um estudo realizado por psiquiatras e professores da Universidade de Singapura apontou para o fato de que o ambiente educacional é um dos fatores que influenciam as escolhas e a maior ou menor receptividade do acadêmico em relação a especialidades médicas. 0 estudo identificou percepções de estudantes de medicina sobre o ambiente de um rodízio prático de psiquiatria e o nível de influência que o ambiente educacional iria exercer sobre a escolha da especialidade dos alunos. Observou-se que os alunos ficaram interessados na eficácia do tratamento realizado pela psiquiatria, melhorando as atitudes em relação à especialidade, mas o estigma da psiquiatria como profissão de sobrecarga mental negativa, muito subjetiva e pouco científica, inferior a outras especialidades e que lida com pacientes perigosos e agressivos continuou um fator de influência negativa sobre a imagem da profissão, algo que é pouco abordado no currículo médico. Concluiu que são necessários mais estudos e intervenções práticas nessa área (Mahendran et al., 2015).

A definição de transtornos mentais de uma forma exclusivamente biológica, além de limitar o indivíduo e transmitir a imagem de um paciente fisiologicamente e geneticamente anormal ou portador de distúrbios apenas neuroquímicos, pode resultar em um impacto social negativo e a uma desumanização, com identificação do sujeito apenas como uma patologia psiquiátrica que necessita de um tratamento farmacológico exclusivo. Assim, a exclusão e o estigma são características negativas presentes em um momento em que o distúrbio psiquiátrico é uma forma de rótulo e transfere uma imagem de loucura associada à anormalidade apenas biológica (Lebowitz \& Ahn, 2014).

Fundado em 2002, o Projeto Icarus foi criado por um grupo de pessoas com diagnóstico de transtorno bipolar que se autoavaliaram como detentoras de características diferentes, que precisam ser cuidadas e aceitas, não devendo ser tratadas como portadoras de uma doença que precisa de cura. O projeto procura enaltecer a diversidade psíquica e uma nova forma de falar sobre saúde mental, não com aspecto de temor, mas como uma parte da personalidade de um indivíduo que possui potenciais criativos e perspectivas de futuro. Definem a sociedade como reprimida e danificada e se veem como pessoas incompreendidas e com histórico de perseguição e estigma social. O projeto possui um site que funciona como suporte, auxílio e interação entre pacientes com transtorno bipolar (DuBrul, 2014).

A necessidade de criar um padrão para o reconhecimento e tratamento do sofrimento psíquico estimulou a Associação Psiquiátrica Americana a criar a primeira edição do DSM, na tentativa de ampliar o entendimento das patologias que surgiam. As definições foram amplamente difundidas e utilizadas como auxílio no maior e melhor diagnóstico e tratamento, buscando atender de forma mais focal e precisa o tratamento da sintomatologia do paciente. Entretanto a dispersão e a fragmentação do sofrimento psíquico foram consequências negativas. Além da ampliação de rotulações e falsas percepções negativas sobre os pacientes com transtorno metal, ocorreram formações de imagens estereotipadas e estigmatizadas também dos profissionais de saúde mental que lidavam com esses pacientes (Assis \& Matthes, 2014; Catani, 2014).

No contexto histórico neoliberal europeu, existem cinco episódios que representaram a perseguição e a crueldade realizada contra indivíduos que possuíam transtornos mentais: a caça às bruxas, a lobotomia, os hospitais-prisões soviéticos, o confinamento e a degeneração e eugenia, em que cada episódio possui diferentes 
modelos conceituais. A lobotomia era uma técnica que interrompia conexões da substância branca dos lobos frontais com o sistema límbico, através do uso do leucótomo. A técnica se popularizou e chegou a ser usada em adolescentes e crianças considerados rebeldes, assim como em oposição política (StucchiPortocarrero, 2013).

Também é importante destacar que o papel da família é essencial no processo de tratamento do indivíduo em sofrimento psíquico, porém familiares também sofrem estigma por serem associados ao transtorno mental do paciente. Esse estigma sofrido por membros da família engloba ideias de contaminação, culpa e vergonha. A ideia de contaminação reflete o quanto a aproximação do indivíduo estigmatizado pode resultar em consequências negativas no imaginário preconceituoso, levando ao aumento da exclusão não apenas do paciente, mas dos familiares que cuidam desses indivíduos (Sousa, 2012; Larson \& Corrigan, 2008).

\section{Objetivos}

Analisar e debater sobre a percepção do acadêmico de medicina da Universidade do Grande Rio, Barra da Tijuca, sobre a atuação da psiquiatria, o ensino da saúde mental na formação médica, o paciente em sofrimento psíquico e outros temas de saúde mental.

\section{Métodos}

Trata-se de um trabalho de natureza aplicada, descritivo, quali-quantitativo, de abordagem teóricoempírica, com pesquisa bibliográfica utilizando a Biblioteca Virtual em Saúde (BVS). É apresentada no presente trabalho uma revisão de literatura envolvendo o tema da percepção da sociedade, com foco em profissionais de saúde e estudantes, frente ao convívio e ao atendimento do paciente psiquiátrico, assim como ao conhecimento de síndromes e diagnósticos nosológicos em psiquiatria, tratamentos e abordagens em saúde mental e do processo de construção histórica das bases da psiquiatria moderna e da evolução da saúde mental, desde a psiquiatria tradicional até as mudanças ocorridas com a Reforma Psiquiátrica. É evidenciada a influência do contexto histórico-cultural e das conquistas obtidas com a luta-antimanicomial no desenvolvimento da psiquiatria moderna, do impacto da persistência de valores, ideias e estereótipos antigos na abordagem, convívio e contato com o paciente psiquiátrico. Os conceitos abordados no trabalho foram: medo do contato com paciente com transtorno mental, carga horária destinada à saúde mental na escola médica, abordagem do paciente e realização de anamnese e exame do estado mental, curto tempo de contato do estudante com a saúde mental, sensação de capacidade para atender portadores de transtornos mentais, interface entre autonomia do paciente e autonomia do exercício profissional médico, conhecimento de referência e de lugares para o tratamento de pacientes com transtornos mentais, ambiente e aspectos de trabalho da psiquiatria, terapias integrativas, modelo de tratamento do paciente com transtorno mental. Os descritores utilizados em língua portuguesa foram percepção, psiquiatria e saúde mental, com saldo de 588 artigos científicos. Foram filtrados posteriormente apenas artigos de língua portuguesa, inglesa e espanhola, selecionando 39 artigos para o presente trabalho, no período de 1985 a 2020.

\section{Amostra}

Foram convidados a participar do presente projeto alunos do curso de Medicina da Unigranrio (Unidade - Barra da Tijuca): do primeiro período, por estarem entrando recentemente em contato com a vida acadêmica no curso de Medicina; do sexto período, por estarem na metade do curso médico; do nono período, que estivessem acabando de sair do rodízio da disciplina do Internato de Psiquiatria. Aqueles que aceitaram fazer parte deste estudo manifestaram sua concordância com a assinatura do TCLE (Termo de Consentimento Livre e Esclarecido).

Em um primeiro momento, a pesquisa foi baseada em um questionário estruturado autoaplicável, abordando conceitos relacionados à saúde mental, selecionados e definidos a partir de estudo da literatura, e evidenciados como focos de atuação dentro das escolas médicas. As perguntas do 
questionário englobavam percepções e sensações de despreparo para o atendimento de pacientes com transtornos mentais, medo do primeiro contato e da continuidade do atendimento desses pacientes, o conhecimento de práticas integrativas e formas terapêuticas para os pacientes, necessidade de encaminhamento de pacientes para avaliação da psiquiatria a partir da atenção básica em saúde, modelo de tratamento empregado ao paciente com transtorno mental, relações de poder e autonomia na relação do psiquiatra com o paciente, papel da figura do hospital psiquiátrico e noções de boa assistência de saúde ligadas ou não à ampliação de leitos vinculados ao modelo manicomialista e visão do próprio estudante de medicina quando este, muitas vezes alvo de gatilhos dentro da própria escola médica, adoece, sofre com sobrecarga física e mental e muitas vezes possui um transtorno mental ou desenvolve ao longo do curso.

A opção de usar um questionário autoaplicável ocorreu para envolver questões, opiniões, percepções e informações dos entrevistados e posteriormente realizar uma análise dos dados obtidos com os aspectos pontuados no instrumento, dirigidos ao grupo de participantes. O questionário utilizado é fruto de uma construção de perguntas elaboradas após estudo bibliográfico e problematização de temas importantes a serem discutidos na educação médica.

O trabalho teve início após submissão do projeto à Plataforma Brasil, tendo respaldo frente ao Comitê de Ética, não possuindo fins lucrativos, não envolvendo pagamento para os entrevistados ou para a universidade. Aprovado pelo Comitê de Ética com CAAE 79510717.0.0000.5283.

\section{Resultados}

Fizeram parte da amostra 133 estudantes de medicina brasileiros de uma universidade privada do Rio de Janeiro, na Barra da Tijuca, sendo 61 deles alunos do primeiro período, 42 do sexto período e 30 do nono período do curso de Medicina. Assim, a população do estudo englobou alunos do ciclo básico, profissionalizante e do internato, representando assim o perfil do acadêmico que está finalizando o primeiro período; englobou alunos que estão saindo do sexto período, alunos na metade do curso de Medicina que já estavam pagando a matéria de Prática Médica, além de englobar os alunos do internato que estariam finalizando o nono período, onde acabaram de sair de um contato ampliado, teórico e prático, tendo como cenários a Colônia Juliano Moreira, englobando o hospital Jurandyr Manfredini e o Instituto de Psiquiatria Philippe Pinel. Inicialmente foi realizado um pré-teste para saber se haveria alguma dúvida em relação às perguntas presentes no questionário. Posteriormente, aplicouse o questionário com esses três grupos. Quanto à variável sexo, 93 estudantes eram do sexo feminino e 40 estudantes do sexo masculino.

Inicialmente, foram analisadas de forma individual as frequências simples de cada uma das 17 perguntas, por porcentagem geral, por sexo do estudante e por semestre do curso de Medicina.

Foi abordado o primeiro conceito de medo do paciente em abordar o transtorno mental. 0 sentimento de medo do estudante de Medicina em relação ao primeiro contato com o paciente com transtorno mental. Ao analisar o perfil de respostas à pergunta 2, levando em consideração o período do curso de Medicina em que o aluno se encontrada, com 4 Graus de liberdade $(d f=4)$, obtivemos um qui-quadrado bastante significante de 0,000 . Tal resultado mostra que o período letivo do estudante ao longo do curso de Medicina influi para a presença ou ausência de medo do primeiro contato com um paciente da psiquiatria. Dos alunos que responderam sim, o menor percentual foi do 1 o período, com $23,9 \%$. 38\% dos alunos que disseram já ter medo do contato com o paciente psiquiátrico eram do 60 período e os outros $38 \%$ restantes são do 9o período. Dos alunos que disseram não ter medo do primeiro contato com esse paciente em nenhum momento da vida, apenas $9,7 \%$ eram do 9o período, enquanto $32,3 \%$ são do 6 o período e a grande maioria, correspondente a $58,1 \%$, é do 1 으 período, antes de um contato efetivo com o paciente psiquiátrico ou com a teoria da saúde mental. Porém, dos 61 estudantes do 1 o período que responderam a essa pergunta, 26 alunos marcaram a opção de que ainda não tiveram a oportunidade do contato com o 
paciente psiquiátrico e não pensaram, portanto, nas possibilidades de interações que viessem a despertar ou não o medo.

Foi abordado o conceito de carga horária destinada à saúde mental questionado se o estudante achava satisfatória a carga horária do curso médico destinada à saúde mental.

Quando considerado o período do curso de Medicina do aluno, o resultado do qui-quadrado foi muito significante, com valor de 0,000. Assim, o período em que o aluno se encontra interfere na percepção em relação a achar satisfatória ou não a carga horária da saúde mental. Evidencia-se que entre o grupo de estudantes que marcou não achar satisfatória a carga horária destinada à saúde mental na formação médica, o maior percentual, correspondendo a $39,2 \%$, são do 6 o período. $32 \%$ são do 1 o período, enquanto os $28,9 \%$ restantes são do 9 o período. Entre os alunos de um mesmo período que responderam ao questionário: $50,8 \%$ do total de alunos do primeiro período que respondeu ao questionário $(n=61)$ indicaram não achar satisfatória a carga horária do curso de Medicina voltada para a saúde mental, $34,4 \%$ achou satisfatória e 14,8\% dos alunos não responderam. Já levando em consideração o número total de alunos do 6 período que responderam ao questionário $(n=42), 90,5 \%$ não acha satisfatória a carga horária da saúde mental na formação do estudante de medicina. No 9o período, 93,3\% não acha satisfatória a carga horária de saúde mental na educação médica.

Foi abordado o conceito anamnese e exame do estado mental questionado se o estudante conhecia a diferença entre anamnese e exame psíquico. Analisando o período em que o aluno se encontrava, obtiveram-se os seguintes resultados: Qui-quadrado significante de 0,000 considerando o período do aluno, para 2 graus de liberdade $(d=2)$. Assim, pode-se afirmar que o período em que o aluno se encontra influencia no conhecimento sobre a diferença teórica e conceitual entre anamnese e exame psíquico. Do total de alunos que responderam ao questionário, $22,6 \%$ responderam sim e são do 9 으 período, apontando para um maior conhecimento teórico sobre o tema, uma vez que a disciplina de psiquiatria é incluída nesse período na faculdade,
16,5\% responderam sim e são do sexto período e $12 \%$ responderam sim e são do primeiro período. $33,8 \%$ do total responderam não e são do primeiro período, mostrando que o maior desconhecimento teórico sobre o tema está no grupo que se encontra no ciclo básico, iniciando a faculdade e com pouco ou quase nulo conhecimento sobre a psiquiatria e saúde mental. $15 \%$ do total responderam não e são do 6 o período e nenhum estudante do 9o período respondeu desconhecer a diferença entre anamnese e exame psíquico.

Foi abordado o conceito tempo de contato com a saúde mental na escola médica questionado se o contato com a saúde mental na formação médica deveria ser ampliado. 94\% do total de estudantes que aceitaram participar da pesquisa (125 alunos) responderam sim e apenas 3,8\% (5 alunos) responderam não achar que o contato com a saúde mental na educação médica deva ser ampliado. 2,3\% (3 alunos) não responderam a essa questão.

Levando em consideração para análise o período do acadêmico de medicina, $39,8 \%$ do total de alunos que responderam ao questionário marcaram sim na pergunta 5 e eram do 10 período. $31,6 \%$ marcaram sim e eram do 6 \% período. Os outros $22,6 \%$ eram de alunos que marcaram sim e eram do 9 o período. $3,8 \%$ do total marcaram não e eram do 1 o período. $0 \%$ responderam não e eram do 6 은 período. $0 \%$ responderam não e eram do 9 o período. Dos que responderam sim, 42,4\% são do 10 período, $33,6 \%$ são do 6 o período e $24 \%$ são do 9 ํ período. Dos 5 alunos que responderam não todos eram do primeiro período, assim como os 3 alunos que não responderam ao questionário, mostrando que nenhum aluno do ciclo clínico entrevistado acha que o contato com a saúde mental dentro da faculdade de medicina não deva ser ampliado. Qui-quadrado significante de 0,040 , considerando o período do aluno. 2 Graus de liberdade ( $d f=2$ ).

Abordou-se o conceito de sensação de capacidade para atender portadores de transtornos mentais. Dos 133 alunos que responderam ao questionário, 7,6\% (10 alunos) afirmaram se sentirem preparados para atender um paciente psiquiátrico, enquanto 92,4\% (123 alunos) não se sentem preparados para atender esse tipo de paciente. 
Dentre os alunos que afirmaram despreparo, 69,9\% eram mulheres e $30,1 \%$ eram homens.

Considerando o período do aluno, 41,4\% afirmaram não estarem preparados e serem do 1 으 período, 30,1\% afirmaram não estarem preparados e serem do 6 o período enquanto $21,1 \%$ disseram não estarem preparados e serem do 9o período. Considerando apenas os alunos do 10 período, apenas $9,8 \%$ se consideravam preparados para atender um paciente psiquiátrico, enquanto $90,2 \%$ não se consideravam aptos a isso. Tal fato é compreendido por estarem no início do ciclo básico, em um primeiro momento e contato com matérias que não englobam a área da saúde mental. Porém, analisando os outros perfis de respostas e comparando, tais resultados com os alunos do sexto período, podemos observar que a maioria dos alunos também se considera despreparada para atender o paciente com transtorno mental, mesmo este estudante se encontrando na metade do curso de Medicina, em termos de períodos percorridos até a formatura.

Ao continuar uma análise ampliada e fazer um comparativo com os alunos do internato, do 9o período, que acabam de pagar a matéria de psiquiatria em um rodízio de 2 meses, observa-se que a grande maioria dos alunos continua se sentindo despreparada para abordar esse tipo de paciente. A pesquisa mostra que $95,2 \%$ dos alunos do 6 ㅇ período sente um despreparo frente o atendimento do paciente psiquiátrico. Do 9o período, 93,3\% dos alunos também não se acham preparados para atender um paciente com transtornos mentais.

Questionou-se ao estudante se o mesmo já deixou de encaminhar algum paciente com suspeita de transtorno mental. De um total de 133 alunos que responderam ao questionário, $31,6 \%$ afirmaram já terem deixado de encaminhar um paciente com suspeita de transtorno mental, enquanto $63,9 \%$ afirmaram nunca terem deixado de fazer o encaminhamento. Apenas $4,5 \%$ do total não respondeu a esta pergunta.

Tendo uma visão ampliada das respostas dos alunos dos 3 períodos, dos que marcaram ter deixado de encaminhar esses pacientes, $47,6 \%$ eram alunos do 9o período, $45,2 \%$ eram alunos do 6 o período e apenas $7,1 \%$ do 10 período. Tal observação mostra que na medida em que se avança no período de medicina o aluno adquire conhecimento e reconhece - subdiagnóstico de pacientes psiquiátricos, refletindo sobre as vezes em que deixou ou não de encaminhar um paciente da área, após o estudo mais ampliado e um conhecimento maior da psiquiatria. Percebe-se também que a maioria dos estudantes de medicina do 9 o período já deixou de encaminhar um paciente da psiquiatria. O cálculo do qui-quadrado se mostrou extremamente significante de 0,000 quando considerado o período do estudante, para 4 graus de liberdade $(\mathrm{df}=4)$. Assim, o período em que o aluno se encontra interfere na percepção dele em relação ao fato de ter deixado ou não de encaminhar algum paciente com suspeita de transtorno mental em algum momento das atividades práticas do curso de Medicina.

Foi abordado o conceito de autonomia do paciente e autonomia do exercício profissional da psiquiatria. Questionou-se ao estudante se ele acreditava que o psiquiatra deveria ter pleno poder para realizar a internação compulsória sem critérios pré-estabelecidos. De um total de 133 alunos respondentes, 78,9\% (105 estudantes) afirmaram que não, que a internação compulsória deve ser realizada pela psiquiatria apenas com critérios estabelecidos, porém ainda existiu um percentual de $20,3 \%$ (27 estudantes) de alunos que acreditam que a internação compulsória deve ser uma decisão do psiquiatra sem a presença de critérios, tendo este plenos poderes sobre a liberdade do paciente e a ordem de internação compulsória.

Foi abordado o conceito dos lugares para tratamento de pacientes com transtornos mentais questionado ao aluno se ele acreditava que existiam poucos hospitais psiquiátricos na cidade do Rio de Janeiro. $77,4 \%$ do total de alunos (103 estudantes) que responderam ao questionário afirmaram achar que o município do Rio de Janeiro possuía poucos hospitais psiquiátricos. Apenas $18 \%$ dos alunos ( 24 estudantes) respondeu não achar o número de hospitais psiquiátricos pequeno e $4,5 \%$ dos alunos ( 6 estudantes) não respondeu a essa pergunta.

Considerando o período do aluno, dentre os estudantes que responderam achar que a cidade do 
Rio de Janeiro possui poucos hospitais psiquiátricos, $43,7 \%$ são do primeiro período, $36,9 \%$ são do 6 ㅇ período e $19,4 \%$ são do 9o período. Observando apenas as respostas dos alunos do primeiro período, $73,8 \%$ responderam sim, enquanto apenas $18 \%$ responderam não. $8,2 \%$ não responderam à pergunta. Em relação apenas aos alunos do 60 período, obteve-se o maior percentual de respostas afirmativas à pergunta, em que $90,5 \%$ mostraram achar que a cidade possui um número reduzido de hospitais psiquiátricos. Entre os alunos do 9o período, $66,7 \%$ responderam sim e $41,7 \%$ responderam não. Qui-quadrado significante de 0,019 considerando o período do aluno, para 4 graus de liberdade $(\mathrm{df}=4)$.

Dentro da abordagem do conceito dos lugares para tratamento de pacientes com transtornos mentais, foi questionado se o estudante acreditava que a ampliação de leitos em hospitais psiquiátricos poderia melhorar a assistência em psiquiatria. Em um total de 133 participantes da pesquisa, $88 \%$ (117 estudantes) responderam acreditar que a melhoria da assistência em psiquiatria estaria ligada ao aumento do número de leitos em hospitais psiquiátricos, enquanto apenas 11,3\% (15 estudantes) responderam não. Um único estudante não respondeu à pergunta.

Analisando as respostas a pergunta $11 \mathrm{e}$ levando em conta o período no curso de Medicina, $40,6 \%$ do total de alunos responderam sim e são do 1 o período, $30,8 \%$ responderam sim e são do 60 período, enquanto $16,5 \%$ responderam sim e são do 9 período. Apenas 1 aluno não respondeu à questão.

O cálculo do qui-quadrado se mostrou significante, com um valor de 0,020 , para 4 graus de liberdade $(\mathrm{df}=4)$, considerando o período dos alunos, mostrando haver relação entre o período em que o aluno se encontra e o padrão de resposta à presente questão.

Em relação a abordagem do conceito sobre o trabalho do psiquiatra, foi questionado se o aluno acha pesado ou carregado o ambiente de trabalho da psiquiatria. De um total de 113 respondentes, $86,4 \%$ dos estudantes de medicina acham pesado ou carregado o ambiente de trabalho da psiquiatria, enquanto apenas $13,6 \%$ não acham.
Levando em conta o período do aluno, $34,1 \%$ dos estudantes responderam sim e são do 1 으 período, 31,1\% responderam sim e são do 60 período, enquanto $21,1 \%$ responderam $\operatorname{sim}$ e são do 9응 período. Entre os que responderam achar o ambiente de trabalho da psiquiatria pesado ou carregado, a maioria é composta de alunos do 10 período, com $39,5 \%$ do total de alunos que responderam sim, ficando o 6 ㅇ período com $36 \%$ e o 9 período com um percentual de 24,6\%. Considerando apenas os alunos do 6 o período, $97,6 \%$ responderam sim, enquanto apenas $2,4 \%$ responderam não achar o ambiente de trabalho da psiquiatria pesado ou carregado. Observando-se apenas os alunos do 9o período (internato), 93,3\% responderam achar o ambiente de trabalho da psiquiatria pesado ou carregado, mesmo tendo passado pela matéria de psiquiatria no internato médico.

Qui-quadrado extremamente significante de 0,02 considerando o período do estudante, para 2 graus de liberdade ( $d f=2)$.

Sobre o conceito de terapias integrativas, foi questionado se o aluno conhecia o trabalho da arteterapia. De um total de 133 estudantes respondentes, apenas $37,6 \%$ dos alunos afirmaram conhecer o trabalho de arteterapia, enquanto a maioria correspondendo a $62,4 \%$ dos alunos afirmaram desconhecer o trabalho.

Considerando o período do curso de Medicina, $10,5 \%$ responderam $\operatorname{sim}$ e são do 10 período, 9\% responderam sim e são do 60 período e $18 \%$ responderam sim e são do 9 o período. $35,3 \%$ responderam não e são do 10 período, $22,6 \%$ responderam não e são do 6ำ período, enquanto apenas 4,5\% responderam não e são do 9 o período. Do total de alunos que afirmaram conhecer o trabalho da arteterapia, a maioria está no 9ㅇ período, correspondendo a $48 \%$ dos estudantes que marcaram sim.

Ainda analisando apenas os estudantes que responderam $\operatorname{sim}, 28,6 \%$ são do 6 o período, enquanto o restante (23\%) é do 1 o período. Quiquadrado extremamente significante de 0,000 considerando o período dos estudantes, para 2 graus de liberdade $(\mathrm{df}=2)$. 
Sobre o conceito de lugares de tratamento, questionou-se o fato de os estudantes saberem o que seria uma Residência Terapêutica (RT). Dos 133 alunos respondentes, apenas 43 alunos (32,3\%) afirmaram saber o significado de uma Residência Terapêutica (RT), enquanto 90 alunos (67\%) afirmaram desconhecer tal significado.

Levando em consideração o período do acadêmico no curso de Medicina, 58,9\% afirmaram não conhecer o significado das residências terapêuticas e ser do 1 - período, enquanto $41,1 \%$ afirmaram não conhecer o significado e ser do 60 período. Nenhum aluno do 9o período demonstrou desconhecer o conceito, afirmando mais uma vez o conhecimento de um aspecto teórico do programa de saúde mental, porém tal fato contrasta com a ideia hospitalocêntrica e curativa tradicionalmente vinculada ao tratamento em ambiente hospitalar da psiquatria, associado ao aumento do número de leitos para uma melhoria da assistência em saúde mental. Qui-quadrado extremamente significante de 0,000 considerando período do estudante, para 2 graus de liberdade $(d f=2)$, mostrando grande relação entre o período em que o aluno se encontra e seu conhecimento sobre o significado teórico das Residências Terapêuticas.

Sobre o conceito modelo de tratamento, questiona-se se o estudante acha que o melhor tratamento para pacientes com transtorno mental seria a prescrição exclusiva de psicofármacos. Dentre os 133 alunos que responderam à questão, 91\% (121 alunos) responderam que não, 1 aluno não respondeu à pergunta, porém ainda existiu um percentual de $8,3 \%$ que respondeu acreditar que a prescrição exclusiva de medicamentos seria o melhor tratamento para um paciente em sofrimento psíquico.

\section{Discussão}

O despreparo de estudantes de medicina e de outros profissionais da área de saúde mental, de acordo com artigos recentemente publicados, relacionam-se a uma carência geral da área de saúde em relação ao sentimento de preparo dos estudantes e diretamente influenciados pela questão de ser o paciente um indivíduo portador de uma doença social e historicamente estigmatizante, além da falta de uma abordagem diferenciada trabalhada de forma correta ou com a carga horária necessária pela educação médica e de outros cursos da área de saúde no Brasil.

É evidenciada no trabalho a importância da vivência teórica e prática adquirida na formação médica, nos ciclos básico e clínico, para a compreensão de formas terapêuticas em saúde mental. Todavia, observa-se que no 9 o período o conhecimento teórico é maior comparado aos outros períodos de referência, porém os alunos ainda possuem uma visão hospitalocêntrica e tradicional da psiquiatria, voltada à assistência em regime de hospitais psiquiátricos e desconhecimento geral das terapias integrativas como formas respaldadas e essenciais para a abordagem do paciente com transtorno mental. Esses alunos também, apesar de terem pago a disciplina, não se sentem preparados para atender esses pacientes, mostrando as várias contradições existentes frente aos desafios não superados pela Reforma Psiquiátrica dentro da formação dos estudantes de medicina.

Ao analisar individualmente o conceito modelos de tratamento, com base nos resultados encontrados apenas no padrão de respostas do 9 ㅇ período, todos os alunos afirmaram não acreditarem que o tratamento exclusivo com psicofármacos seja o melhor tratamento para pacientes com transtorno mental, evidenciando que um dos pontos-chave da luta antimanicomial e da Reforma Psiquiátrica está totalmente enraizado na mente dos estudantes de medicina do espaço amostral, que estão no internato médico, não estando a imagem da especialidade ligada apenas a de um trabalho essencialmente voltado a prescrição medicamentosa na percepção dos alunos do internato, especificamente os do 9o período.

O maior percentual de alunos que cursam um período específico e responderam acreditar que a ampliação do número de leitos em hospitais psiquiátricos seria uma alternativa para melhorar a assistência em psiquiatria é do 1ำ período (ciclo básico), que possui o menor contato com a saúde mental, porém outros acadêmicos no meio do curso de Medicina e do internato também mostraram 
acreditar a existência de uma relação da boa assistência em saúde mental com a hospitalização e ampliação de leitos. Analisando apenas as respostas dos alunos do 9o período, que estariam mais próximos da formatura, observa-se que a maioria dos estudantes (73,3\% do total de alunos do período) acredita que aumentar o número de leitos e hospitais psiquiátricos auxiliaria a uma melhor assistência em psiquiatria, mais uma vez reforçando que o modelo médico tradicional hospitalocêntrico e manicomialista ainda é forte, mesmo entre alunos do internato que já passaram pela disciplina de Psiquiatria, evidenciando para a realidade local da universidade analisada o que a literatura já aponta como desafio da Reforma Psiquiátrica que ainda não foi totalmente superado, tendo grandes raízes no ensino médico e expandindo com maiores proporções para a saúde quando esse perfil de estudante adentra no mercado de trabalho e atua como médico formado, generalista.

\section{Conclusão}

O fato de existirem estigmas, preconceitos e estereótipos envolvendo a figura da psiquiatria e do paciente com transtorno mental, imagens negativas historicamente enraizadas e presentes tanto na população geral quanto em médicos e estudantes de medicina deve ser considerado, principalmente ao se falar sobre o assunto da importância de um maior contato e novas formas de inserção e expansão do ensino prático e teórico da saúde mental na educação médica.

É necessário a busca de reformulações sobre o ensino médico da saúde mental, através de intervenções específicas na educação que permitam um contato ampliado e mais frequente de alunos com pacientes psiquiátricos e familiares, com um conhecimento claro e amplo dos diversos recursos terapêuticos disponíveis para o atendimento desse paciente nos diferentes níveis de atenção.

A formação de médicos generalistas sem uma visão holística e integral do ser humano afeta diretamente os pacientes em sofrimento psíquico, levando a consequências negativas físicas, mentais, sociais e financeiras, pela elevação de gastos com a saúde individual. Tal problemática é agravada pela inexistência no Brasil de organizações responsáveis pela padronização do ensino da saúde mental nos cursos de medicina, estando essa abordagem restrita às referências contidas em diretrizes curriculares, algo que vem se mostrando ineficiente para suprir as novas necessidades de saúde com a crescente presença de transtornos mentais frequentes em pacientes como desafios a serem enfrentados para correto diagnóstico, abordagem e tratamento pelo médico generalista.

Compreender o paciente é analisar todas as suas esferas de uma forma holística e integrada, com uma visão do mesmo como participante integrado por meio da história de vida e percepção da sua experiência vivida diante do que lhe afeta em toda a sua complexidade. Usando esse ponto de vista como guia, a perpetuação de atitudes estigmatizantes externa e internamente contribui para a fuga social do portador de doença mental pelo medo de revelar sua doença, assim como de comentar abertamente sobre as angústias e problemas que passa, os tratamentos que realiza, além dos conflitos e sentimentos subjetivos que permeiam sua nova realidade, mantendo-a muitas vezes oculta, gerando discriminação e mitos sem fundamentação realística. Observa-se, após a aplicação do questionário e também após o grupo de debates, que, em todos os três períodos, a maioria dos alunos acredita que a cidade do Rio de Janeiro possui poucos hospitais, evidenciando um caráter hospitalocêntrico tradicional associado à ideia de tratamento do paciente com transtornos mentais, presente mesmo em estudantes de períodos mais avançados, incluindo os que estão cursando o internato e já tiveram contato com todas as disciplinas relacionadas a saúde mental, psicologia médica e psiquiatria.

A percepção que estudantes de medicina têm em relação ao paciente com transtorno mental é a de que se trata de um paciente diferenciado, que requer um tratamento distinto e direcionado em relação à gravidade específica do quadro. Acreditam que a figura desse paciente seja definida como de alguém instável, que pode se descontrolar a qualquer momento, com atitudes muitas vezes agressivas, imprevisíveis e duvidosas. Tais estudantes 
em um momento da reflexão admitem poderem estar expressando representações do senso comum, historicamente construídas, e que auxiliam na manutenção das opressões e da marginalização dos pacientes com transtornos mentais. A pesquisa e os debates e a revisão de literatura mostraram que ainda existe uma imagem historicamente imposta a ser superada, inclusive dentro do âmbito da educação médica, devendo essa questão ser trabalhada com os acadêmicos no debate de estereótipos e construção do imaginário da psiquiatria, também como dos motivos que levam a essa percepção da psiquiatria como algo relacionado ao peso e a uma carga subjetiva envolvida, assim como o pensamento de formas de intervenção para mudanças da realidade, além do mapeamento desse padrão em outras escolas médicas do Brasil.

\section{Contribuição}

As pesquisadoras declaram não haver conflitos de interesse.

\section{Referências}

Alverga, A. R., \& Dimenstein, M. (2006). A reforma psiquiátrica e os desafios na desinstitucionalização da loucura. Interface - Comunicação, Saúde, Educação, 10(20), 229-316. https://www.scielo.br/scielo. php?pid=S1414-32832006000200003\&script=sci_ abstract\&tlng=pt

Amarante, P., \& Torres, E. H. G. (2001). A constituição de novas práticas no campo da atenção psicossocial: análise de dois projetos pioneiros na Reforma Psiquiátrica no Brasil. Saúde em Debate, 25(58), 26-34. https://www. arca.fiocruz.br/handle/icict/26183

Antunes, H. M., Campos, C. J. G., Lima, G. M. P. A., \& Ferraz, I. L. G. (2016). Motivos e crenças de familiares frente ao tratamento do transtorno depressivo na infância: Estudo qualitativo. Estudos de Psicologia, 21(1), 157-166. https://www.scielo.br/scielo.php?script=sci abstract\&pid=S1413-294X2016000200157\&Ing $=e n \& n r m=i s o \& t \operatorname{lng}=p t$
Araruna, M. H. M., Filha, M. O. F., Dias, M. D., Braga, L. A. V., Moraes, M. N., \& Rocha, I. A. (2012). Formação de terapeutas comunitários na Paraíba: impacto na Estratégia Saúde da Família. Enfermagem: Revista Eletrônica, 14(1), 33-41. https://revistas.ufg.br/fen/article/view/15679

Assis, C. L., \& Matthes, G. A. S. (2014).

Representações sociais sobre a psicologia e o psicólogo em universitários de uma faculdade privada de Rondônia, Brasil. Aletheia, 43(44), 66-90. http://www.periodicos.ulbra.br/index. php/aletheia/article/view/3300

Benedetto, M. A. C., \& Gallian, D. M. C. (2018). Narrativas de Estudantes de Medicina e Enfermagem: currículo oculto e desumanização em saúde. Interface (Botucatu), 22(67), 1197-1207. https:// www.scielo.br/j/icse/a/ WHPJt7wnscmbY Bt7dhL76ZD/?lang=pt\&format=pdf

Brockelman, K. F., \& Scheyett, A. M. (2015). Faculty perceptions of accommodations, strategies, and psychiatric advance directives for university students with mental illnesses. Psychiatric Rehabilitation Journal, 38(4), 342-348. https:// pubmed.ncbi.nlm.nih.gov/26053532/

Camargo, I. B., \& Contel, J. O. B. (2004). Tradução e adaptação de questionários norteamericanos para a avaliação de habilidades e conhecimentos na prática psiquiátrica brasileira. Revista de Psiquiatria do Rio Grande do Sul, 26(3), 288-299. https:// www.scielo.br/scielo.php?script=sci arttext \&pid=S0101-81082004000300006

Castro, R. C. B. R., \& Silva, M. J. P. (2002). 0 conhecimento e a percepção do enfermeiro a respeito do processo da reforma psiquiátrica. Acta Paulista de Enfermagem, 15(2), 55-64. https://acta-ape.org/article/o-conhecimentoe-a-percepcao-do-enfermeiro-a-respeito-doprocesso-da-reforma-psiquiatrica

Catani, J. (2014). Histeria, transtornos somatoformes e sintomas somáticos: as múltiplas configurações do sofrimento psíquico no interior dos sistemas classificatórios. Jornal de Psicanálise, 47(86), 115-134. http://pepsic. bvsalud.org/scielo.php?script=sci_ arttext\&pid=S0103-58352014000100012 
Cyrino, E. G., \& Toralles-Pereira, M. L. (2004).

Trabalhando com estratégias de ensinoaprendizado por descoberta na área da saúde: a problematização e a aprendizagem baseada em problemas. Cadernos de Saúde Pública, 20(3), 780-788. https://www.scielo. $\mathrm{br} / \mathrm{scielo}$.php?script=sci_arttext\&pid=S0102311X2004000300015

Daltro, M. R., \& Pondé, M. P. (2011). Atenção psicopedagógica no ensino superior: uma experiência inovadora na graduação de medicina. Construção Psicopedagógica, 19(18), 104-123. http:// pepsic.bvsalud.org/scielo.php?script=sci_ arttext\&pid=S1415-69542011000100010

Dâmaso, J. G. B., Pereira, B. S., Batista, C. B., Conceição, L. S., Pereira, G. S., \& Carniele, R. C. (2019). É muita pressão! Percepções sobre o desgaste mental entre os estudantes de medicina. Revista Brasileira de Orientação Profissional, 20(2), 29-41. http://pepsic. bvsalud.org/pdf/rbop/v20n2/a04v20n2.pdf

DuBrul, S. A. (2014). The Icarus Project: A Counter Narrative for Psychic Diversity. Journal of Medical Humanities, 35(3), 257-271. https:// pubmed.ncbi.nlm.nih.gov/25030378/

Feijó, L. P., Motta, S. G., Saldanha, R. P., Kubrusly, M., \& Augusto, K. L. (2019). Revista Brasileira de Educação Médica, 43(4), 141-150. https:// www.scielo.br/j/rbem/a/MQ73rWHGwm8 CNvdfYPNznRR/?lang=pt\&format=pdf

Ferreira, C. M. G., Kluthcovsky, A. C. G. C., \& Cordeiro, T. M. G. (2016). Prevalência de Transtornos Mentais Comuns e Fatores Associados em Estudantes de Medicina: um Estudo Comparativo. Revista Brasileira de Educação Médica, 40(2), 268-277. https://www.scielo.br/ scielo.php?pid=S0100-550220160002002 $68 \&$ script $=$ sci_abstract $\&$ tlng $=p t$

Ferreira-Furegato, A. R., Frari-Galera, S. A., Pillon, S. C., Ferreira-Santos, J. L., Araujo-Pitia, A. C., \& Cardoso, L. (2010). Characterizing mental healthcare service teams. Revista de Salud Pública, 12(5), 732-743. http:// www.scielo.org.co/scielo.php?script=sci arttext\&pid=S0124-00642010000500004
Firmin, M. W., Wantz, R. A., Geib, E. F., \& Ray, B. N. (2012). Undergraduate Students' Perceptions of Practicing Psychiatrists. Journal of Psychiatric Practice, 18(6), 460-466. https://journals.Iww. com/practicalpsychiatry/Abstract/2012/11000/ Undergraduate_Students_Perceptions_of_ Practicing.11.aspx

Gonçalves, M. B., \& Benevides-Pereira, A. M. T. (2009). Considerações sobre o ensino médico no Brasil: consequências afetivo-emocionais nos estudantes. Revista Brasileira de Educação Médica, 33(3), 493-504. https://www.scielo. $\mathrm{br} / \mathrm{scielo}$.php?script=sci_arttext\&pid=S010055022009000300020\&lng=en\&nrm=iso\&tlng=pt

Larson, J. E., \& Corrigan, P. (2008). The Stigma of Families with Mental Illness. Academic Psychiatry, 32(2), 87-91. https://pubmed.ncbi. nlm.nih.gov/18349326

Lebowitz, M. S., \& Ahn, W.K. (2014). Effects of biological explanations for mental disorders on clinicians' empathy. Psychological and Cognitive Sciences, 111(50), 17786-17790. https:// pubmed.ncbi.nlm.nih.gov/25453068

Lima, A. C. C., Caponi, S., \& Minella, L. S. (2010). Reflexões sobre as disputas das comunidades científicas pela regulação do campo psi. Malestar e Subjetividade. 10(1), 37-60. http:// pepsic.bvsalud.org/scielo.php?script=sci arttext\&pid=S1518-61482010000100003

Mahendran, R., Lim, H. A., Verma, S., \& Kua, E. $H$. (2015). The impact of the educational environment on career choice and attitudes toward psychiatry. Medical Teacher, 37(5), 494-497. https://pubmed.ncbi.nlm.nih. gov/25693795

Marco, O. L. N. M. (2009). O estudante de Medicina e a procura de ajuda. Revista Brasileira de Educação Médica, 33(3), 487-492. https:// www.scielo.br/scielo.php?script=sci arttext\&pid=S0100-55022009000300019

Mascia, A. R., \& Silva, F. B., Lucchese, A. C., De Marco, M. A., Martins, M. C. F. N., \& Martins, L. A. N. (2009). Atitudes frente a aspectos relevantes da prática médica: estudo transversal randomizado com alunos de segundo e sexto anos. Revista Brasileira de Educação Médica, 33(1), 40-48. https://www.scielo.br/scielo.php?pid=S0100$55022009000100006 \&$ script=sci_abstract\&tlng=pt 
Martinhago, F., \& Oliveira, W.F. (2015). (Des) institucionalização: a percepção dos profissionais dos Centros de Atenção Psicossocial de Santa Catarina. Saúde e Sociedade, 24(4), 1273-1284. https:// www.scielo.br/scielo.php?pid=S0104$12902015000401273 \&$ script $=$ sci_ abstract\&tlng=pt

Otanari, T. M. C., Leal, E. M., Campos, R. T. O., Palombini, A. L. P., \& Pereira, E. H. P. (2011). Os efeitos na formação de residentes de psiquiatria ao experimentarem grupos GAM. Revista Brasileira de Educação Médica, 35(4), 460-467. Recuperado de: https:// www.scielo.br/scielo.php?pid=S0100$55022011000400004 \&$ script $=$ sci_ abstract\&tlng=pt

Paulin, L. F. R. S., \& Poças, R. C. G. (2009). A experiência da Universidade São Francisco com o internato médico de psiquiatria utilizando a metodologia da aprendizagem baseada em problemas. Revista de Psiquiatria do Rio Grande do Sul, 31(1), 67-72. https://doi. org/10.1590/S0101-81082009000100012.

Pereira, A. A., Costa, A. N., \& Megale, R. F. (2012). Saúde mental para médicos que atuam na estratégia saúde da família: uma contribuição sobre o processo de formação em serviço. Revista Brasileira de Educação Médica, 36(2), 269-279. https:// www.scielo.br/scielo.php?pid=S0100$55022012000400017 \&$ script $=$ sci abstract\&tlng=pt

Rocha, G. D. S. C., Barcelos, I. C. R. R., \& Coloda, R. G. (2010). A Distância da Proximidade entre o Asilo Psiquiátrico e a Produção de Sentidos: O Aprendizado de Enfermagem Psiquiátrica nos Dias Atuais. Revista de Pesquisa Cuidado é Fundamental, 2(4), 1313-1325. https:// www.redalyc.org/pdf/5057/Resumenes/ Resumen_505750833031_1.pdf

Sakellari, E., Lehtonen, K., Sourander, A., KalokerinouAnagnostopoulou, A., \& Leino-Kilpi, H. (2014). Greek adolescents' views of people with mental illness through drawings: mental health education's impact. Nursing and Health Sciences, 16(3), 358-364. https://onlinelibrary.wiley.com/ doi/abs/10.1111/nhs.12113
Santos, V. H., Ferreira, J. H., Alves, G. C. A., Naves, N. M., Oliveira, S. L., Raimondi, G. A., \& Paulino, D. B. (2020). Currículo oculto, educação médica e profissionalismo: uma revisão integrativa. Interface (Botucatu), 24, 1-17. https://www.scielo.br/j/icse/a/ hCDK4YXNhzJxfPX7FBXj3Lc/?lang=pt

Shackle, E.M. (1985). Psychiatric diagnosis as an ethical problem. J Med Ethics, 11(3), 132-134. https://www.ncbi.nlm.nih.gov/pmc/articles/ PMC1375175/

Soares, A. N., Silveira, B. V., Santos, F. B. O., Alves, P. N. M., \& Lana, F. C. F. (2013). (Re)significando os espaços assistenciais em saúde mental: relato de experiência. Texto Contexto \& Enfermagem, 22(4), 1214-1222. https://www.scielo.br/scielo. php?pid=S0104-07072013000400041\&script=sci_ abstract\&tlng=pt.

Sousa, P. F., Maciel, S. C., Medeiros, K. T., \& Vieira, G. L. S. (2016). Atitudes e Representações em saúde mental: um estudo com universitários. Psico-USF, 21(3), 527-538. https://pesquisa. bvsalud.org/portal/resource/pt/biblio-829354

Sousa, S., Marques, A., Curral, R., \& Queirós, C. (2012). Stigmatizing attitudes in relatives of people with schizophrenia: a study using the Attribution Questionnaire AQ -27. Trends in Psychiatry and Psychotherapy, 34(4), 186-197. https://repositorio-aberto.up.pt/ bitstream/10216/65356/2/87747.pdf

Souza, C. P. J., \& Lima, F. M. L. S. (2016). O Papel da Psicoterapia de Grupo na Formação do Residente em Psiquiatria. Revista Brasileira de Educação Médica, 40(1), 109-117. https:// www.scielo.br/scielo.php?pid=S0100$55022016000100109 \&$ script $=$ sci abstract\&tlng=pt

Stucchi-Portocarrero, S. (2013). Estigma, discriminación y concepto de enfermedad mental. Revista de Neuropsiquiatria, 76(4), 218-223. https://revistas.upch.edu.pe/index. php/RNP/article/view/1170

Touso, M. F. S., \& Figueiredo, G. L. A. (2020). A educação médica nos cenários de práticas em anos iniciais da formação: sentidos tecidos pelo photovoice. Interface (Botucatu), 24, 1-19. https://www.scielo. $\mathrm{br} / \mathrm{j} / \mathrm{icse} / \mathrm{a} / \mathrm{tmKM} 3 \mathrm{xscytSc9pfdYjh89fG}$ /?format=pdf\&lang=pt 